\title{
Proteasome-independent p53 degradation
}

\author{
Cell Research (2013) 23:597-598. doi:10.1038/cr.2013.38; published online 12 March 2013
}

The intracellular levels of the p53 tumor suppressor protein are regulated through various pathways and involve numerous regulatory components. A recent study published in Cell Research identifies a proteasomeindependent pathway of $\mathrm{p} 53$ protein degradation in the nucleolus that is dependent on Def and Calpain3.

The $\mathrm{p} 53$ tumor suppressor protein is a central player in the cellular response to stress. In response to diverse stress signals including DNA damage, nutrient deprivation and oncogene activation, p53 protein becomes activated and functions as a sequence-specific DNAbinding transcription factor to regulate the expression of both protein-coding and -noncoding genes that collectively result in cell cycle arrest, senescence or apoptosis. p53 proteins levels are kept low through various pathways that promote $\mathrm{p} 53$ protein degradation including: (i) the ubiquitin-mediated $26 \mathrm{~S}$ proteasome-dependent pathway, catalyzed by a number of different E3 ubiquitin ligases including Mdm2, Pirh2 and COP1 (reviewed in [1]); (ii) the 20S proteasome-dependent "degradation by default" pathway for intrinsically unstructured proteins (reviewed in [2]); and (iii) the cytoplasmic calpaindependent $\mathrm{p} 53$ protein degradation pathway [3-5].

In a recent study published in Cell Research, Tao et al. [6] reveal a new conserved nucleolar pathway for $\mathrm{p} 53$ protein turnover which is dependent on the digestive organ expansion factor (Def) protein and the nucleolar cysteine protease Calpain3. Def protein was first characterized in zebrafish as an endoderm-specific factor important for digestive organ expansion and growth [7]. Homozygous deletion of the def gene in zebrafish, the defiti29 mutant, resulted in the arrest of cell proliferation and impaired organ growth in a number of digestive organs including the liver, the exocrine pancreas and the gallbladder. The deffitz29 mutants subsequently die at 8-11 days post-fertilization [7]. The gene expression profiles of wildtype and $d e^{f i t a 29}$ mutant zebrafish were compared and this revealed that expression of p53 responsive genes including Mdm2, p21 and cyclin G1 were upregulated in the defini29 mutant [7]. p53 transcript levels, however, were not elevated in the mutant, indicating that the increased expression of $\mathrm{p} 53$ target genes may be due to increase in $\mathrm{p} 53$ protein levels or activity $[6,7]$. Based on these results, Tao et al. [6] further show that Def is localized to the nucleolus in both human cell lines and zebrafish. Using both wild-type and $d e f^{\text {hit2 } 29}$ zebrafish, as well as an array of def-specific morpholino antisense oligonucleotides and siRNA, Tao et al. [6] demonstrate that $\mathrm{p} 53$ protein accumulates in the nucleolus upon silencing of the def gene, and that re-introduction of def through mRNA expression constructs results in $\mathrm{p} 53$ protein degradation, both in the presence and absence of $\mathrm{Mdm} 2$, the key E3 ligase implicated in $\mathrm{p} 53$ protein degradation. Using inhibitors that target cysteine proteases, the lysosome and the $26 \mathrm{~S}$ and $20 \mathrm{~S}$ proteasomes, the authors identify a cysteine protease, Calpain3, as the protease likely involved in Defmediated p53 degradation and show that Calpain3 is capable of forming a complex with Def independently of p53, and that Calpain 3 enzymatic activity is crucial for $\mathrm{p} 53$ protein turnover. Finally, Tao et al. [6] suggest a model whereby Def may act as a scaffold to recruit the Calpain 3 protease, p53 and perhaps other unidentified effectors to regulate p53 protein levels in the nucleolus. Accordingly, p53-mediated responses of cell cycle arrest and apoptosis are blocked, and cell proliferation and differentiation can proceed unhindered during organogenesis and development.

Results from this study are notable as they identify a mechanism for nucleolar p53 protein turnover. This is important for at least two reasons. First, it contributes to our understanding of p53 degradation through Mdm2- and proteasome-independent pathways and second, it highlights the possibility that p53 may have nucleolar-specific functions. The nucleolus serves as the site for rRNA transcription, processing and ribosome assembly. Nucleoli are also implicated as sensors of various forms of cellular stress that impair ribosomal biogenesis $[8,9]$. During nucleolar stress (also known as ribosomal stress), several ribosomal proteins including RPL5, RPL11 and RPL23 bind to $\mathrm{Mdm} 2$ and inhibit its E3 ligase function, leading to $\mathrm{p} 53$ stabilization and activation in the nucleus. This is reminiscent of the action of the nucleolar protein, p14 ARF, that binds and sequesters $\mathrm{Mdm} 2$ in the nucleolus in response to oncogenic stress. Elevated levels of p53 protein in nucleoli are observed in response to proteasome inhibition with MG132 [10,11]. The function of $\mathrm{p} 53$ in the nucleolus is uncertain but nucleolar localization may facilitate the ability of $\mathrm{p} 53$ to repress RNA polymerase 1 activity which is required for synthesis 
of $47 \mathrm{~S}$ precursor rRNA transcripts that are processed to form $18 \mathrm{~S}, 5.8 \mathrm{~S}$ and 28S rRNAs [11]. The importance of def during embryonic development in fish suggests that nucleolar p53 needs to be tightly regulated so that it is available to regulate ribosome biogenesis at times of stress without interfering with normal embryonic development under normal stress-free conditions.

The study by Tao et al. [6] delineates yet another pathway for control of p53 protein turnover and raises a number of intriguing questions: (i) What phenotype is exhibited by def-deficient mice? Do def-deficient mice exhibit elevated levels of p53 and do they exhibit impaired digestive tract development as seen in zebrafish? Is this phenotype rescued in a $d e f^{--} p 53^{-/}$double mutant? (ii) Is the muscular dystrophy phenotype seen in Calpain3-knockout mice due to unrestrained p53 protein activity [12]?
If so, would a Calpain $3^{-/} p 53^{-/}$double knockout mouse have a rescued phenotype and would a $d e f^{-/}$mouse resemble a Calpain $3^{--}$mouse? (iii) What physiological signals govern activation of the Calpain3-Def complex in vivo and how does this pathway impact p53 cellular functions? (iv) What other substrates are targeted for degradation by the DefCalpain3 nucleolar complex in vivo? The answers to these questions will help further define the regulation of p53 protein turnover in the cell and introduce yet another layer of complexity in the regulation of p53 function.

\section{Mais M Nuaaman' ${ }^{1}$, Samuel Benchimol ${ }^{1}$}

${ }^{1}$ Department of Biology, York University, Toronto, ON M3J 1P3, Canada Correspondence: Samuel Benchimol

E-mail: benchimo@yorku.ca

\section{References}

1 Tai E, Benchimol S. Proc Natl Acad Sci USA 2009; 106:11431-11432.

2 Tsvetkov P, Reuven N, Shaul Y. Cell Death Differ 2010; 17:103-108.

3 Kubbutat MHG, Vousden KH. Mol Cell Biol 1997; 17:460-468

4 Pariat M, Carillo S, Molinari M, et al. $\mathrm{Mol}$ Cell Biol 1997; 17:2806-2815.

5 Gonen H, Shkedy D, Barnoy S, et al. FEBS Lett 1997; 406:17-22.

6 Tao T, Shi H, Guan Y, et al. Cell Res 2013; 23:620-634.

7 Chen J, Ruan H, Ng SM, et al. Genes Dev 2005; 19:2900-2911.

8 Rubbi CP, Milner J. EMBO J2003; 22:60686077.

9 Zhang Y, Lu H. Cancer Cell 2009; 16:369377.

10 Klibanov SA, O’Hagan HM, Ljungman M. J Cell Sci 2001; 114:1867-1873.

11 Karni-Schmidt O, Zupnick A, Castillo M, et al. J Cell Sci 2008; 121:4098-4105.

12 Richard I, Broux O, Allamand V, et al. Cell 1995; 81:27-40. 